Article

\title{
Some Intrinsic Properties of Tadmor-Tanner Functions: Related Problems and Possible Applications
}

\author{
Nikolay Kyurkchiev
}

Faculty of Mathematics and Informatics, University of Plovdiv Paisii Hilendarski, 24, Tzar Asen Str., 4000 Plovdiv, Bulgaria; nkyurk@uni-plovdiv.bg

Received: 24 September 2020; Accepted: 12 October 2020; Published: 5 November 2020

\begin{abstract}
In this paper, we study some properties of an exponentially optimal filter proposed by Tadmor and Tanner. More precisely, we consider the problem for approximating the function of rectangular type $F(t)$ by the class of exponential functions $\sigma_{\text {adapt }}(t)$ about the Hausdorff metric. We prove upper and lower estimates for "saturation" $-d$ (in the case $q=2$ ). New activation and "semi-activation" functions based on $\sigma_{\text {adapt }}(t)$ are defined. Some related problems are discussed. We also consider modified families of functions with "polynomial variable transfer". Numerical examples, illustrating our results using CAS MATHEMATICA are given.
\end{abstract}

Keywords: exponentially optimal adaptive filter; Hausdorff distance; upper and lower bounds; activation function; modified families of functions with "polynomial variable transfer"

MSC: 41A46

\section{Introduction}

In antenna-feeder techniques, the most often occurred signals $F(t)$ are of rectangular type, as shown in Figure 1. In [1,2], the authors construct a new class of accurate filters for processing piecewise smooth spectral data.

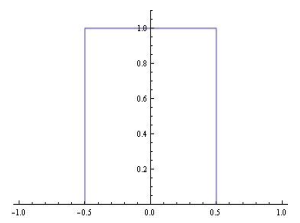

Figure 1. Signal of rectangular type- $F(t)$.

Definition 1. The exponentially optimal filter is defined as [1,2]:

$$
\sigma_{\text {adapt }}(t):=\left\{\begin{array}{l}
e^{\frac{c_{q} q}{t^{2}-1}} ;|t|<1, \quad c_{q}=2^{q} \cdot \frac{3}{8} \cdot \frac{18 q^{2}+3 q+14}{9 q^{2}+6 q+2} . \\
0,|t| \geq 1
\end{array}\right.
$$

Various modifications of this "powerful" class of functions have been proposed and studied by a number of researchers. This study represents a certain interesting problem for approximating the function $F(t)$ with the specified class of exponential functions $\sigma_{\text {adapt }}(t)$ in the Hausdorff sense. 
Definition 2 ([3,4]). The Hausdorff distance (the H-distance) [3] $\rho(f, g)$ between two interval functions $f, g$ on $\Omega \subseteq \mathbb{R}$, is the distance between their completed graphs $F(f)$ and $F(g)$ considered as closed subsets of $\Omega \times \mathbb{R}$. More precisely,

$$
\rho(f, g)=\max \left\{\sup _{A \in F(f)} \inf _{B \in F(g)}\|A-B\|, \sup _{B \in F(g)} \inf _{A \in F(f)}\|A-B\|\right\}
$$

wherein $\|$.$\| is any norm in \mathbb{R}^{2}$-e. g. the maximum norm $\|(t, x)\|=\max \{|t|,|x|\}$-hence, the distance between the points $A=\left(t_{A}, x_{A}\right), B=\left(t_{B}, x_{B}\right)$ in $\mathbb{R}^{2}$ is $\|A-B\|=\max \left(\left|t_{A}-t_{B}\right|,\left|x_{A}-x_{B}\right|\right)$.

The basic approaches for approximation of functions and point sets of the plane by algebraic and trigonometric polynomials in respect to Hausdorff distance (H-distance) are connected to the work and achievements of Bl. Sendov who established a Bulgarian school in Approximation theory, particularly for developing the theory of Hausdorff approximations.

For some basic results, see [5,6].

In this paper we consider some intrinsic properties of function (1). A number of modified adaptive functions have also been proposed that can find application in the field of antenna-feeder analysis. Of course, the specialists working in this direction will assess which of the new models have the right to exist.

\section{Main Results}

\subsection{Some Intrinsic Properties}

We will consider only the case $q$-even (see, Figure 2 ). Let $t_{0}$ be the value for which $\sigma_{\text {adapt }}\left(t_{0}\right)=$ $\frac{1}{2}$-i.e., $t_{0}$ is the solution of the nonlinear equation:

$$
e^{\frac{c_{q} t_{0}^{q}}{t_{0}^{2}-1}}-\frac{1}{2}=0
$$

The Hausdorff distance $d$ between $F(t)$ and $\sigma_{\text {adapt }}(t)$ satisfies the relation:

$$
e^{\frac{c_{q}\left(t_{0}-d\right)^{q}}{\left(t_{0}-d\right)^{2}-1}}=1-d
$$

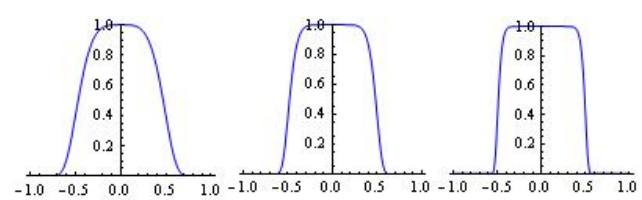

Figure 2. The exponentially optimal filter $\sigma_{\text {adapt }}(t)$ for $q=4,8,16$.

Some computational examples using relations (3) and (4) are depicted on Figure 3. Equations (3) and (4) are nonlinear equations for solving $t_{0}$ and $d$. For large values of $q$, the coefficient $c_{q}$ grows very rapidly, which severely limits the computational calculations and visualization of the results in any Computer Algebraic Platform. The same remark remains valid when solving the nonlinear Equation (4) for the magnitude of the best Hausdorff approximation- $d$. This requires obtaining precise two-sided estimates for $d$. We will sketch the idea, for example, for $q=2$. Let (see (4))

$$
G(d)=c_{2} \frac{\left(t_{0}-d\right)^{2}}{\left(t_{0}-d\right)^{2}-1}-\ln (1-d) .
$$

The following theorem gives upper and lower bounds for $d$. 
Theorem 1. Let $q=2$. The "saturation"-d satisfies the following inequalities

$$
d_{l}:=\frac{1}{2 p_{2}}<d<\frac{\ln \left(2 p_{2}\right)}{2 p_{2}}:=d_{r} .
$$

where

$$
p_{2}=\frac{1+2 c_{2} t_{0}-2 t_{0}^{2}+t_{0}^{4}}{\left(t_{0}^{2}-1\right)^{2}}
$$

Proof. From $G^{\prime}(d)>0$, we conclude that the function $G$ is strictly monotonically increasing. Consider the function

$$
G_{1}(d)=c_{2} \frac{t_{0}^{2}}{t_{0}^{2}-1}+\frac{1+2 c_{2} t_{0}-2 t_{0}^{2}+t_{0}^{4}}{\left(t_{0}^{2}-1\right)^{2}} d=p_{1}+p_{2} d .
$$
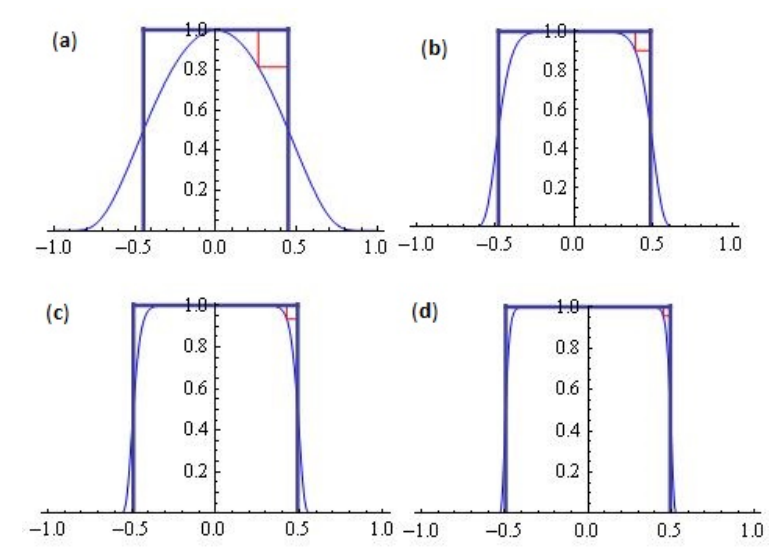

Figure 3. Approximation of $F(t)$ by $\sigma_{\text {adapt }}(t)$ : (a) $q=2, c_{q}=2.76, t_{0}=0.448028$; Hausdorff distance $d=0.185197 ;(\mathbf{b}) q=8, c_{q}=182.492, t_{0}=0.482049$; Hausdorff distance $d=0.0974479 ;$ (c) $q=16, c_{q}=47781, t_{0}=0.489945 ;$ Hausdorff distance $d=0.0646479$; (d) $q=30, c_{q}=7.9266 \times 10^{8}, t_{0}=0.494313 ;$ Hausdorff distance $d=0.0427321$.

From Taylor expansion, we obtain $G(d)-G_{1}(d)=O\left(d^{2}\right)$. Hence, $G_{1}(d)$ approximates $G(d)$ with $d \rightarrow 0$ as $O\left(d^{2}\right)$ (see, Figure 4 ). In addition, $G_{1}^{\prime}(d)>0$. Further, we have

$$
\begin{gathered}
G_{1}\left(\frac{1}{2 p_{2}}\right)=p_{1}+\frac{1}{2}=-0.193147<0 \\
G_{1}\left(\frac{\ln \left(2 p_{2}\right)}{2 p_{2}}\right)=p_{1}+\frac{1}{2} \ln \left(2 p_{2}\right)=0.445106>0 .
\end{gathered}
$$

This completes the proof of the theorem.

From (5), we see that for $q=2, d_{l}=0.102642<d=0.185197<d_{r}=0.233665$.

The reader may formulate the corresponding approximation problem for arbitrary $q$ following the ideas given in this note, and this will be omitted. Some techniques for obtaining accurate two-sided estimates can be found in [7].

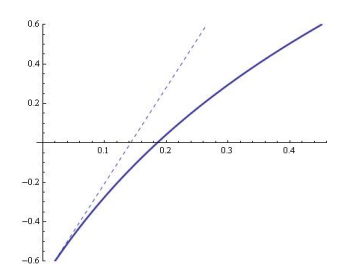

Figure 4. The functions $G(d)$ and $G_{1}(d)$ for $q=2$. 


\subsection{Some Remarks}

The basic problems considered in [8-10]) are an approximation of functions and point sets by algebraic and trigonometric polynomials in the Hausdorff metric as well as their applications in the field of antenna-feeder techniques, analysis and the synthesis of antenna patterns and filters, including noise minimization by the suitable approximation of impulse functions.

The application of Fourier analysis for the approximation of impulse and transmitting functions is compared to this, which can be obtained using the elements of the best Hausdorff approximation.

The advantage of the Hausdoorff metric is the removal of "Gibbs' phenomenon" [11] (see, Figure 5).

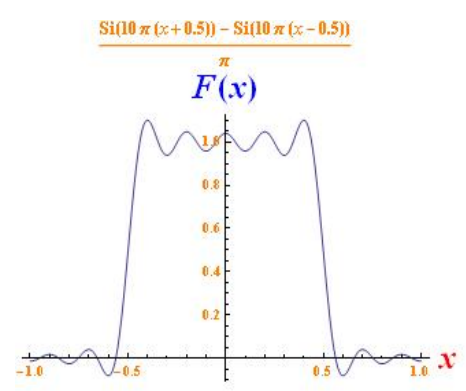

Figure 5. A typical application of Fourier transform to analysis of radiation patterns (“Gibbs' phenomenon") [8].

- Modified Families with "Polynomial Variable Transfer"

Following the ideas formally given by us, we consider the following modified families of functions based on $\sigma_{\text {adapt }}(t)$ with "polynomial variable transfer":

$$
\begin{aligned}
& F_{1}(t):=e^{\frac{c_{q} f(t) q}{t^{2}-1}} \\
& f(t)=\sum_{i=0}^{n} a_{i} t^{i}, \quad a_{0}=0
\end{aligned}
$$

and

$$
\begin{aligned}
& F_{2}(t):=e^{\frac{\left.c_{q} f(t)\right)^{q}}{f(t)^{2}-1}}, \\
& f(t)=\sum_{i=0}^{n} a_{i} t^{i}, \quad a_{0}=0 .
\end{aligned}
$$

The modified families: $F_{1}(t)$ for $q=2, n=3, a_{0}=0, a_{1}=3.96, a_{2}=-18.9, a_{3}=5.7$ and $F_{2}(t)$ for $q=2, n=4, a_{0}=0, a_{1}=-1.96, a_{2}=8.9, a_{3}=-6.7, a_{4}=2$ are depicted in Figure 6 .

Let $t=b_{0}(\pi \cos (\theta)+a)$, where $\theta$ is azimuthal angle and $a$ is the phase difference.

Then, for example, typical radiation patterns with "restrictions" using $F_{1}(\theta)$ for

(a) $q=2, n=3, a_{0}=0, a_{1}=1, a_{2}=-3.9, b_{0}=-0.5, a=3.2$;

(b) $q=2, n=3, a_{0}=0, a_{1}=1, a_{2}=-3.9, b_{0}=-1.8, a=2.4$

are plotted on Figures 7 and 8.

Numerical examples are presented using CAS MATHEMATICA. 

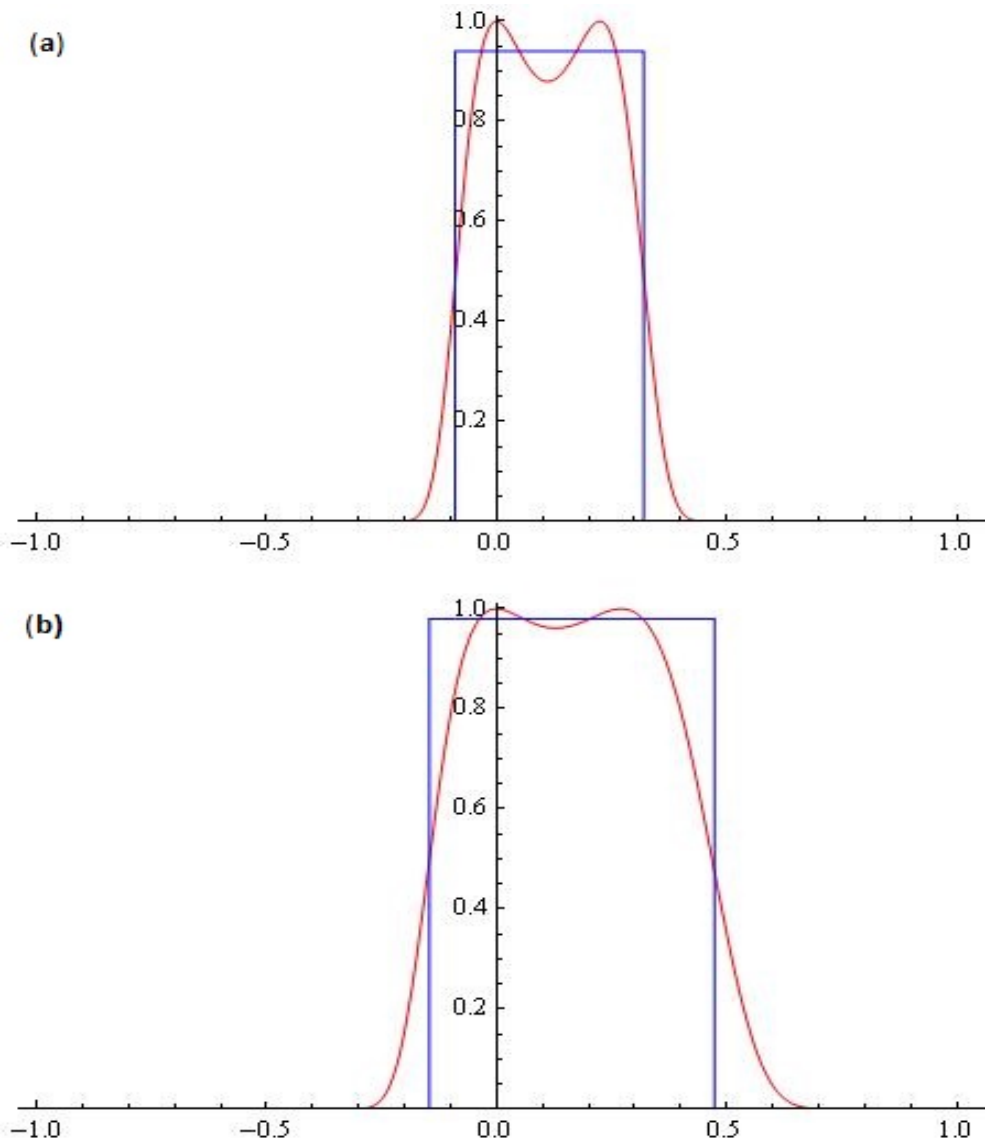

Figure 6. (a) Modified family $F_{1}(t)$ for $q=2, n=3, a_{0}=0, a_{1}=3.96, a_{2}=-18.9, a_{3}=5.7$; (b) Modified family $F_{2}(t)$ for $q=2, n=4, a_{0}=0, a_{1}=-1.96, a_{2}=8.9, a_{3}=-6.7, a_{4}=2$.
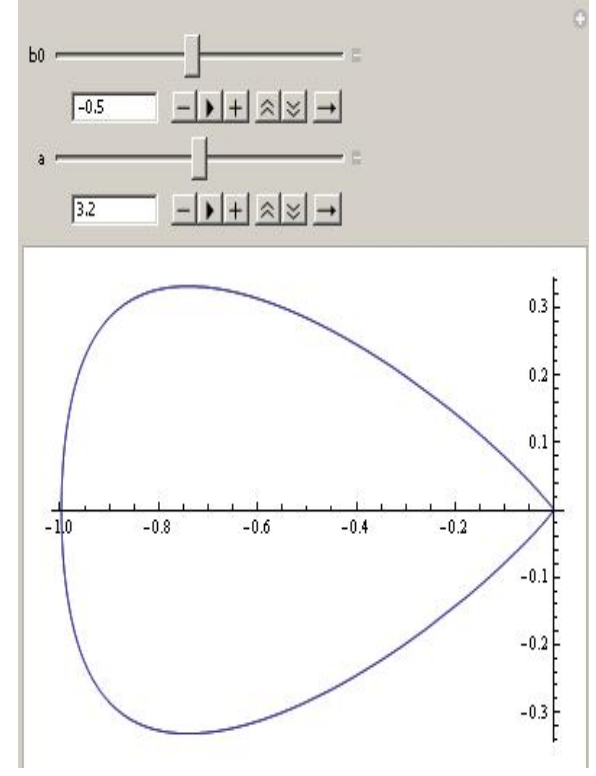

Figure 7. A typical radiation pattern with "restrictions" using $F_{1}(\theta)$ for $q=2, n=3, a_{0}=0, a_{1}=1$, $a_{2}=-3.9, a_{3}=1.7, b_{0}=-0.5, a=3.2$. 


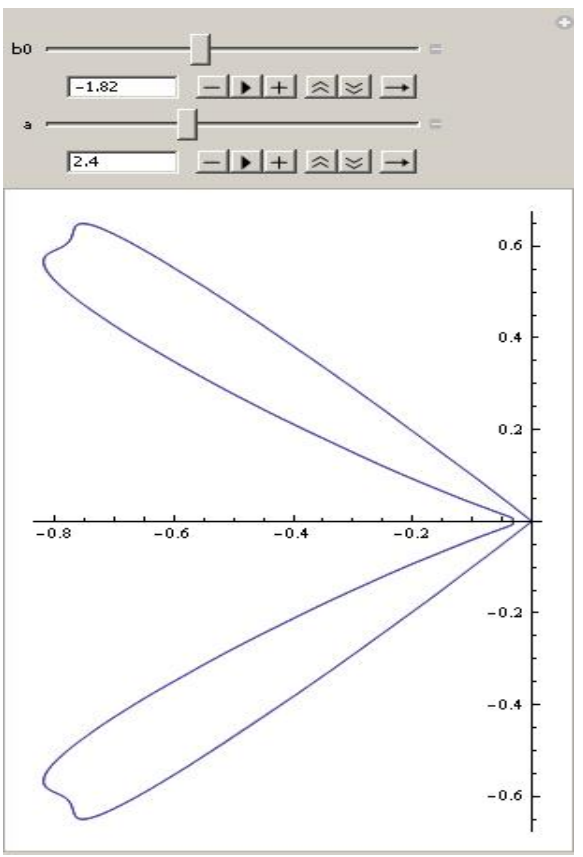

Figure 8. A typical radiation pattern with "restrictions" using $F_{1}(\theta)$ for $q=2, n=3, a_{0}=0, a_{1}=1$, $a_{2}=-3.9, a_{3}=1.7, b_{0}=-1.82, a=2.4$.

- The New Activation Function

We define the following activation function based on $\sigma_{\text {adapt }}(t)(1)$ :

$$
F_{3}(t)=\frac{1-e^{\frac{c_{q} q^{2}}{t^{2}-1}}}{1+e^{\frac{c_{q} q^{2}}{t^{2}-1}}}
$$

In antenna-feeder technique most often occurred signals are of types shown in Figures 9 and 10. For $q$ even, the corresponding approximation using model (9) is shown in Figure 9. For $q$ odd, the corresponding approximation using new activation function $F_{3}(t)$ is shown in Figure 10.
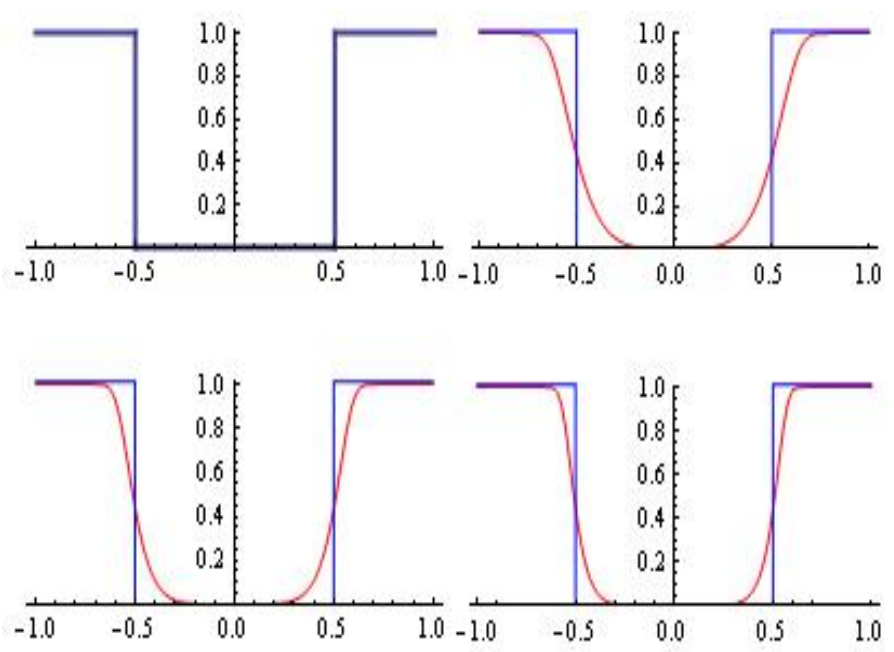

Figure 9. Approximation by $F_{3}(t)$ for $q=4,6,8$. 

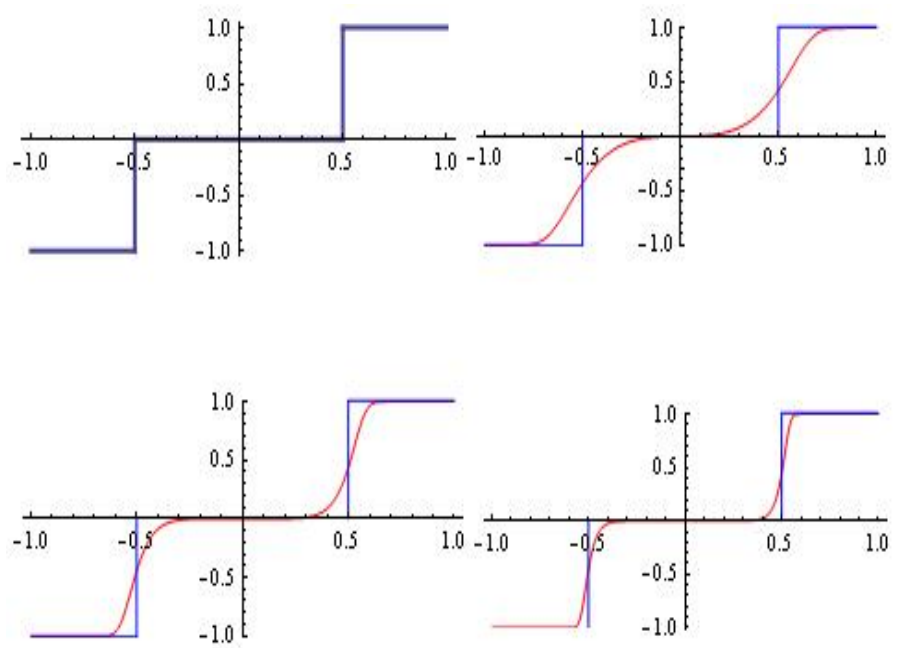

Figure 10. Approximation by $F_{3}(t)$ for $q=3,7,13$.

The reader may formulate the corresponding approximation problem following the ideas given in this article, and this will be omitted. The results have important role in the fields of Population Dynamics, Biostatistics, Signal Theory, Reliability Analysis and Life testing experiments. For other results, see [12-15]. Another application of the Hausdorff metric can be found in [16].

- New "semi-activation" function

We define the following "semi-activation" function

$$
F_{4}(t)=\frac{1-e^{\frac{c_{q} t^{q}}{\left(a_{0}+a_{1} t+a_{2} t^{2}\right)^{2}-1}}}{1+e^{\frac{c_{q}\left(a_{0}+a_{1} t+a_{2} t^{2}\right)^{q}}{\left(a_{0}+a_{1} t+a_{2} t^{2}\right)^{2}-1}}}
$$

The problem of approximating the "point set" depicted in Figure 11 is also of independent interest (in the limiting case, the "point set CROSS"—-see, for instance, [17]).
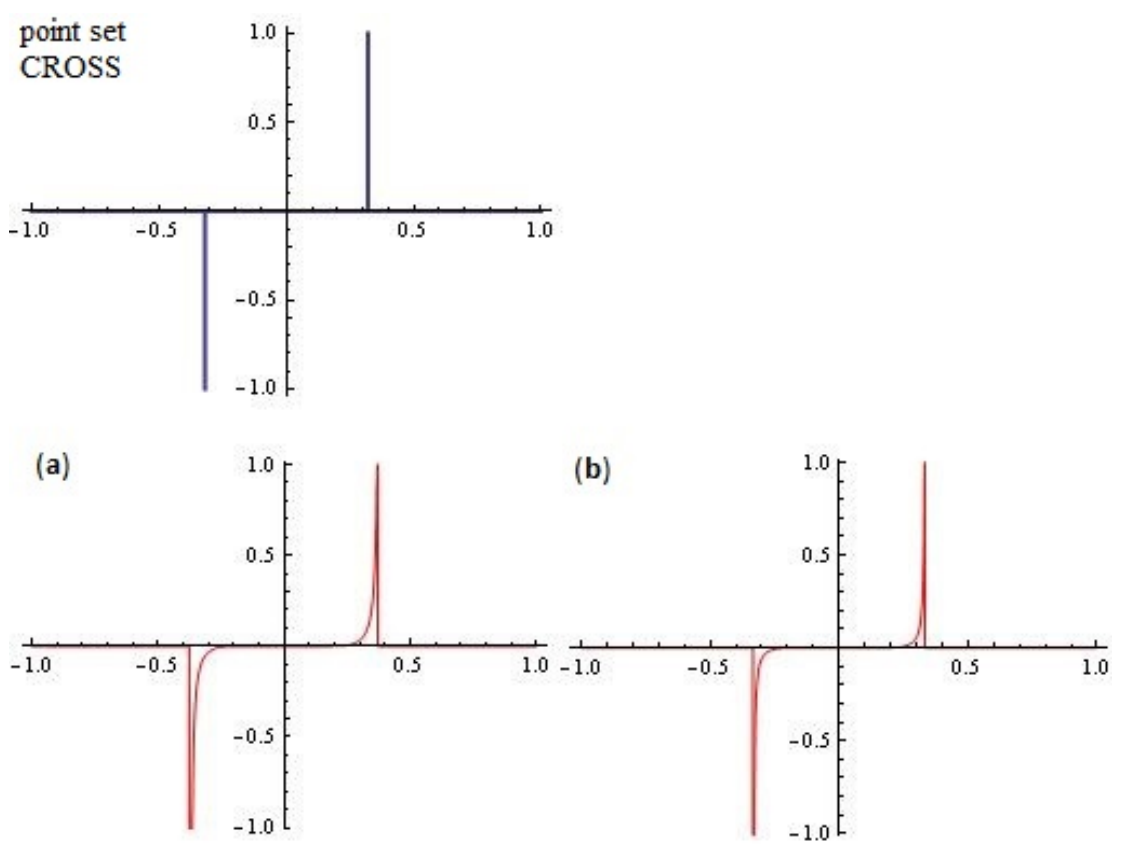

Figure 11. Approximation by $F_{4}(t), q=7$ : (a) $a_{0}=0, a_{1}=0.04, a_{2}=7.2 ;$ (b) $a_{0}=0, a_{1}=0.01, a_{2}=$ 9.01 . 
The modified family $F_{4}(t)$ for $q=7$ :

(a) $a_{0}=0, a_{1}=0.04, a_{2}=7.2$;

(b) $a_{0}=0, a_{1}=0.01, a_{2}=9.01$

is depicted in Figure 11.

- Another activation function

Formally, we define the following activation function

$$
F_{5}(t)=\frac{e^{-\frac{a t^{q}}{t^{2}-1}}-e^{\frac{a t^{q}}{t^{2}-1}}}{e^{-\frac{a t^{q}}{t^{2}-1}}+e^{\frac{a t^{q}}{t^{2}-1}}}, \quad t \in(0.1)
$$

where $a>0$.

The shifted Heaviside step function is defined by

$$
h_{t_{0}}(t)=\left\{\begin{array}{ccc}
0, & \text { if } & t<t_{0} \\
{[0,1],} & \text { if } & t=t_{0} \\
1, & \text { if } & t>t_{0}
\end{array}\right.
$$

Obviously, the new function $F_{5}(t)$ can be used successfully to approximate the Heaviside step function (see Figure 12).
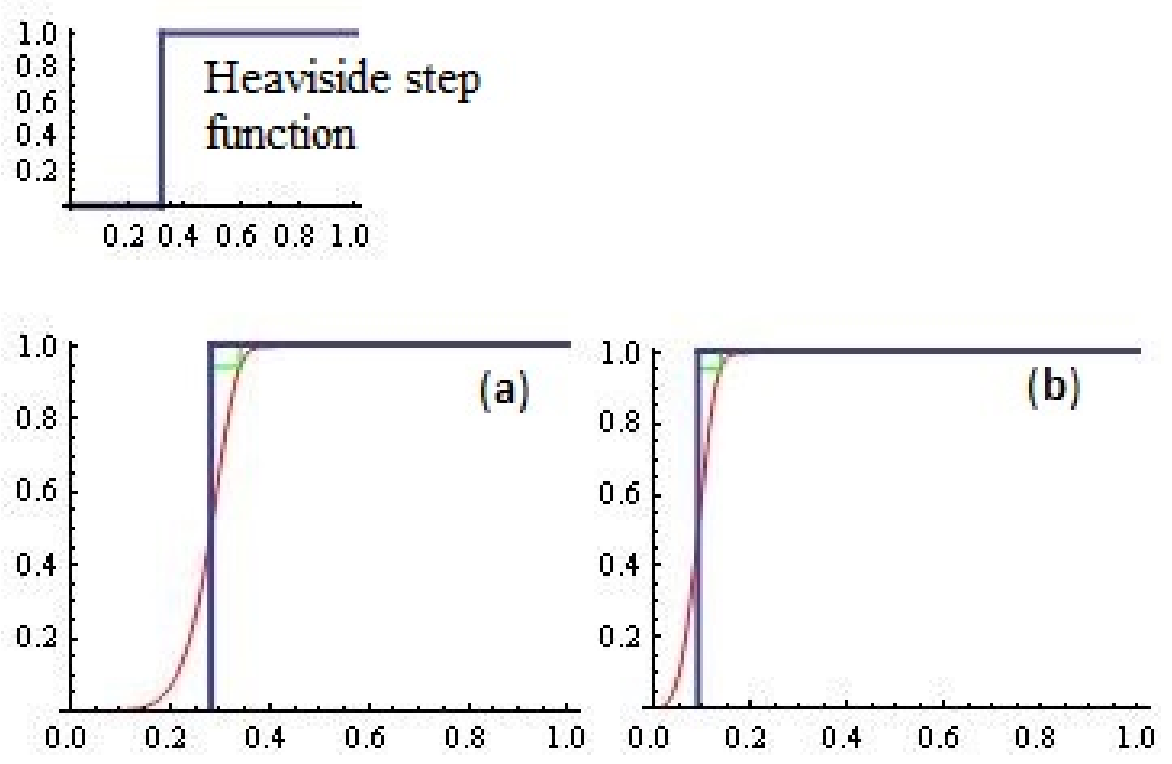

Figure 12. Approximation of $h_{t_{0}}(t)$ by $F_{5}(t):(a) q=6$ : a) $a=1000, t_{0}=0.282245$; Hausdorff distance $d=0.0580217$; (b) $q=3$ : a) $a=700, t_{0}=0.0919762$; Hausdorff distance $d=0.0460022$.

Approximation of shifted Heaviside step function $h_{t_{0}}(t)$ by sigmoidal function $F_{5}(t)$ for:

(a) $q=6: a=1000, t_{0}=0.282245$; Hausdorff distance $d=0.0580217$;

(b) $q=3 a=700, t_{0}=0.0919762$; Hausdorff distance $d=0.0460022$

is visualized on Figure 12.

The function $F_{5}(t)$ can be used in the field of population dynamics and biostatistics [7]. 
- The new function

Formally, we define the following function:

$$
F_{6}(t)=1-e^{-\frac{H(t)}{G(t)}}
$$

where $H(t)$ and $G(t)$ are polynomials.

In some cases, the function $F_{6}(t)$ can be used for analysis of "rectangular signals".

For example, see Figure 13 for fixed

$G(t)=1.17755 t^{10}-51.87041 * t^{9}+2.85984 * t^{8}+123.85465 * t^{7}-14.21173 * t^{6}-100.59484 * t^{5}+$ $16.45178 * t^{4}+30.42548 * t^{3}-7.39353 * t^{2}-0.95663 * t+1.1$ and $H(t)=t^{8}+t^{6}+t^{4}+t^{2}+2.9$.

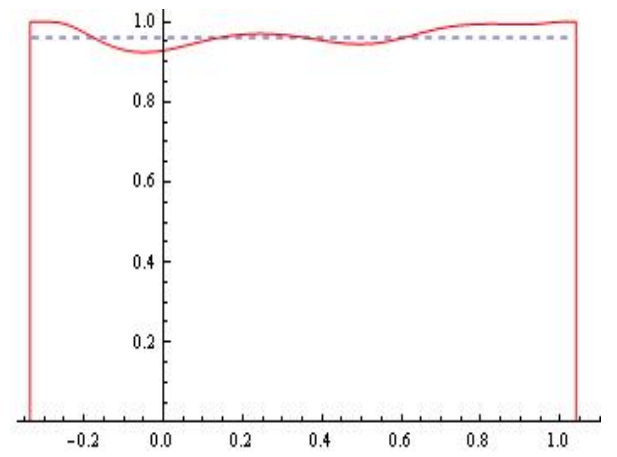

Figure 13. The function $F_{5}(t)$-red.

Remark 1. $G(t)$ is the polynomial of best one-sided Hausdorff distance of the function

$$
\kappa(t)=\left\{\begin{array}{cll}
-1, & \text { if } & -1 \leq t \leq-\frac{1}{3} \\
1, & \text { if } & -\frac{1}{3}<t \leq 1
\end{array}\right.
$$

by polynomial of degree 10 (see, for example, [4]).

Let $t=b_{0}(\pi \cos (\theta)+a)$.

A "typical radiation pattern" $F_{6}(\theta)$ for $b=0.132$ and $a=4.263$ is depicted on Figures 14 and 15. Unfortunately, these diagrams cannot always be realized in practice. 


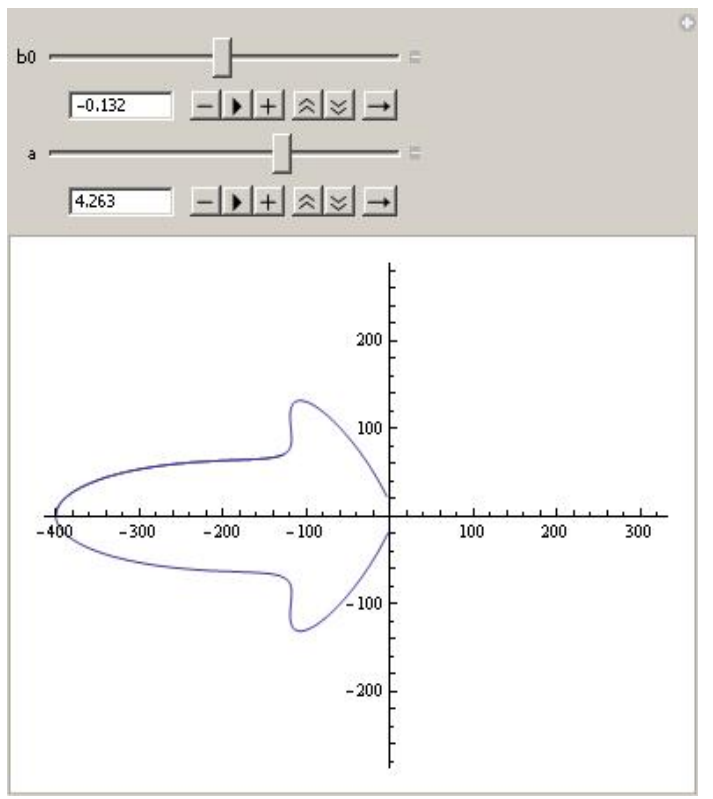

Figure 14. A “typical radiation pattern" $F_{6}(\theta)$ for $b=0.132$ and $a=4.263$.

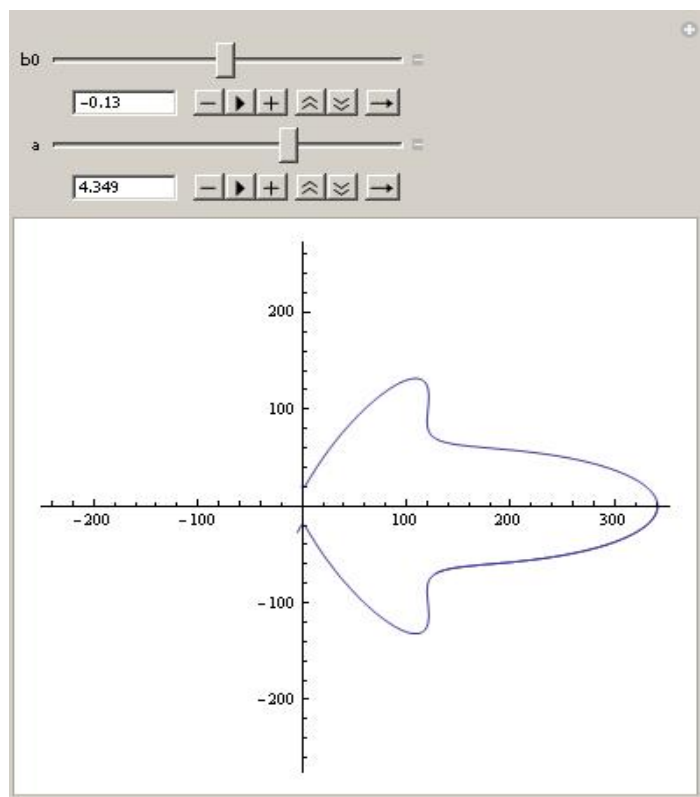

Figure 15. A "typical radiation pattern" $F_{6}(\theta)$ for $b=-0.13$ and $a=4.349$.

- Application to the "cut-off function"

The analysis presented in this article can also be applied to the "cut-off function", see (1):

$$
F_{7}(t)=e^{-\frac{C t^{p}}{t^{2}-\pi^{2}}}
$$

where $C>0$ and $p$ is even.

- Approximation with restrictions

Often some restrictions must be imposed on the main lobe of the radiation pattern-for example, see Figure 16 
The polynomial

$$
f(t)=133.123786 t^{10}-251.561221 t^{8}+107.004415 t^{6}+36.476971 t^{4}-30.804320 t^{2}+6.189054
$$

computed by Remez's algorithm from [18] and shown in Figure 17, provides a reliable approximation of the window (Figure 16).

We note that the "adaptive function" with $c_{1}=6.9$

$$
F_{8}(t)=\frac{1}{\left(1-e^{\frac{c_{1} t^{2}}{2^{2}-1}}\right)^{2}}
$$

based on function of Tadmor and Tanner (1) is of the form shown in Figure 17.

Unfortunately, these diagrams cannot always be realized in practice.

Specialists working in these scientific fields have the final word.

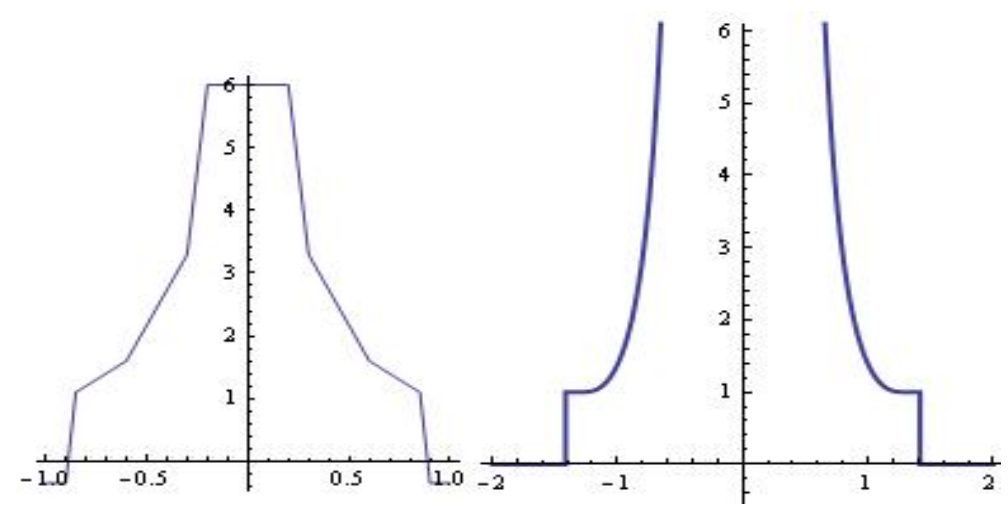

Figure 16. "Typical restrictions".
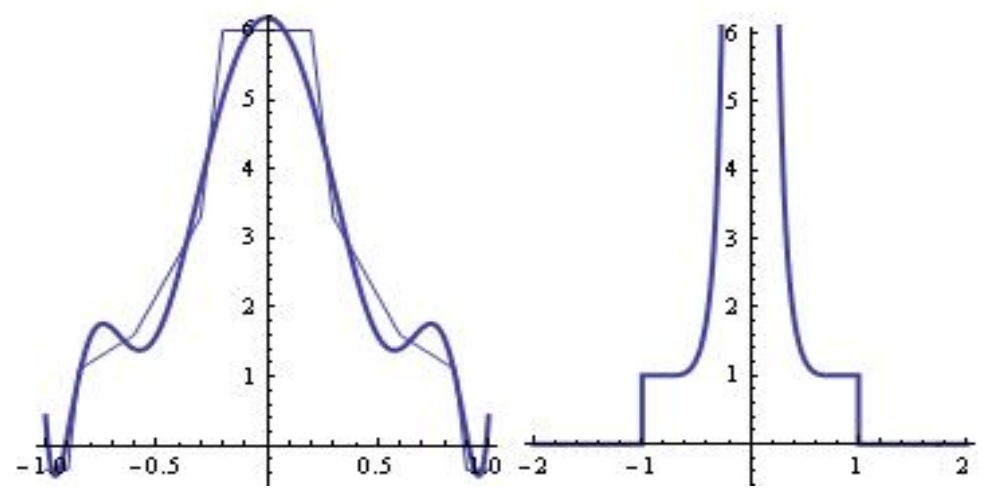

Figure 17. "Typical radiation patterns".

We will look at another instructive example.

A typical diagram function $R(t)$ with imposed constraints at the median level is shown in Figure 18. 


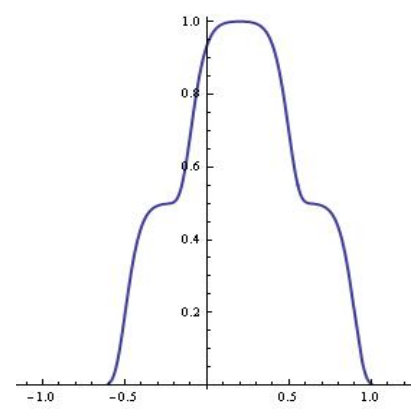

Figure 18. A typical diagram function $R(t)$ with restrictions.

After serious analysis, it turned out that the function by Tadmor and Tanner could be modified in such a way as to obtain the following "differential analogue with step $h$ ":

$$
\sigma_{\text {adapt }}^{*}(t):=\left(\sigma_{\text {adapt }}(t)+\sigma_{\text {adapt }}(t-h)\right) / 2
$$

where

$$
\sigma_{\text {adapt }}(t):=e^{\frac{c_{q} q q}{t^{2}-1}}
$$

and

$$
c_{q}=2^{q} \cdot \frac{3}{8} \cdot \frac{18 q^{2}+3 q+14}{9 q^{2}+6 q+2} .
$$

From the attached visualization (see Figures 19 and 20) it can be seen that the proposed new modification can be used successfully for the approximation of functions of type $R(t)$.

Obviously, higher order differential analogues can be obtained, which we leave to the readers' attention.
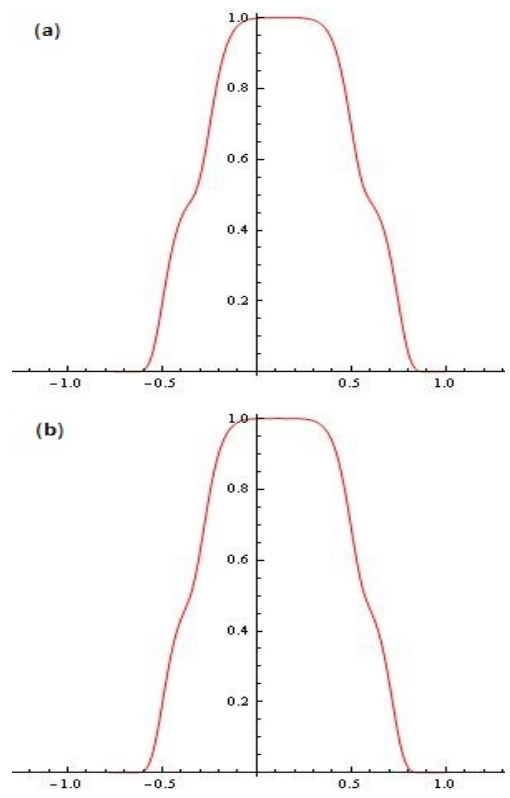

Figure 19. The function $\sigma_{\text {adapt }}^{*}(t)$ for $q=8:(\mathbf{a}) h=0.32 ;(\mathbf{b}) h=0.22$. 

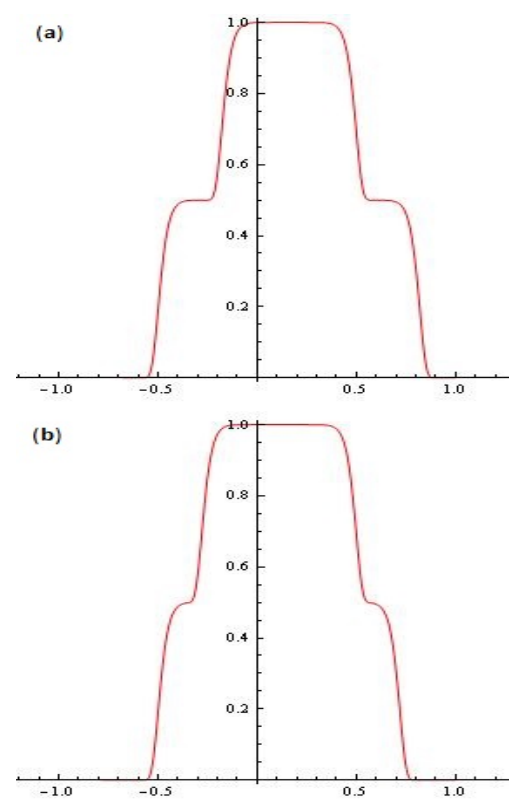

Figure 20. The function $\sigma_{\text {adapt }}^{*}(t)$ for $q=16$ : (a) $h=0.32 ;(\mathbf{b}) h=0.22$.

\section{Concluding Remarks}

The aim of the present research is to define families of "adaptive functions" that could be used in various branches of scientific knowledge, in particular in the approximation of a number of classical impulse signals, point sets in the plane and supplemented graphs of discontinuous functions.

Questions related to the synthesis and analysis of transfer functions, radiation diagrams with algebraic and trigonometric polynomials about Hausdorff distance are elaborated in detail in the monograph in [8].

In this article we consider only some aspects related to the disclosure of intrinsic properties of some of the proposed families of "adaptive functions" in a purely methodological aspect.

Many of the proposed new models could not be used as "feasible models-diagrams" in practice.

In this connection, the specialists working in the indicated field have the floor. For some results, see [19-39].

Funding: This research received no external funding.

Acknowledgments: This work has been accomplished with the financial support by the Grant No BG05M2OP001-1.001-0003, financed by the Science and Education for Smart Growth Operational Program (2014-2020) and co-financed by the European Union through the European structural and Investment funds.

Conflicts of Interest: The author declares no conflict of interest.

\section{References}

1. Tadmor, E.; Tanner, J. Adaptive filters for piecewise smooth spectral data. SIMA J. Numer. Alg. $2005,25,535-547$.

2. Tanner, J. Optimal filter and mollifier for piecewise smooth spectral data. Math. Comput. 2006, 75, 767-790. [CrossRef]

3. Hausdorff, F. Set Theory, 2nd ed.; Chelsea Publ.: New York, NY, USA, 1962.

4. Sendov, B. Hausdorff Approximations; Kluwer: Boston, MA, USA, 1990.

5. Sendov, B.; Popov, V. The exact asymptotic behavior of the best approximation by algebraic and trigonometric polynomials in the Hausdorff metric. Math. USSR-Sb 1972, 89, 138-147. (In Russian) [CrossRef]

6. Anguelov, R.; Markov, S.; Sendov B. On the Normed Linear Space of Hausdorff Continuous Functions. Lecture Notes Comput. Sci. 2006, 3743, 281-288.

7. Kyurkchiev, N.; Markov, S. On the Hausdorff distance between the Heaviside step function and Verhulst logistic function. J. Math. Chem. 2016, 54, 109-119. [CrossRef] 
8. Kyurkchiev, N.; Andreev, A. Approximation and Antenna and Filter Synthesis: Some Moduli in Programming Environment Mathematica; Lap Lambert Academic Publishing: Saarbrucken, Germany, 2014.

9. Andreev, A.; Kyurkchiev, N. Approximation of some impulse functions-implementation in programming environment MATHEMATICA. In Proceedings of the 43 Spring Conference of the Union of Bulgarian Mathematicians, Borovetz, Bulgaria, 2-6 April 2014; pp. 111-117.

10. Golev, A.; Djamiykov, T.; Kyurkchiev, N. Sigmoidal Functions in Antenna-feeder Technique. Int. J. Pure Appl. Math. 2017, 116, 1081-1092.

11. Gelb, A.; Tanner, J. Robust reprojection methods for the resolution of the Gibbs phenomenon. Appl. Comput. Harmon. Anal. 2006, 20, 3-25. [CrossRef]

12. Kyurkchiev, N.; Iliev, A.; Rahnev, A. On the Half-Logistic Model with "polynomial variable transfer". Application to approximate the specific "data CORONA VIRUS". Int. J. Differ. Equations Appl. 2020, 19, 45-61.

13. Kyurkchiev, N. Selected Topics in Mathematical Modeling: Some New Trends (Dedicated to Academician Blagovest Sendov (1932-2020)); Lap Lambert Academic Publishing: Saarbrucken, Germany, 2020.

14. Costarelli, D.; Spigler, R.; Constructive Approximation by Superposition of Sigmoidal Functions. Anal. Theory Appl. 2013 29, 169-196.

15. Kyurkchiev, N.; Nikolov, G. Comments on some new classes of sigmoidal and activation functions. Applications. Dyn. Syst. Appl. 2019, 28, 789-808.

16. Peters, J. Foundations of Computer Vision: Computational Geometry, Visual Image Structures and Object Shape Detection; Springer: Cham, Switzerland, 2017.

17. Kyurkchiev, N.; Markov, S. On the numerical approximation of the "cross" set. Ann. Univ. Sofia Fac. Math. 1974, 66, 19-25. (In Bulgarian)

18. Tashev, S. Approximation of bounded sets on the plane in Hausdorff metric. CR Acad. Bulg. Sci. 1976, 29, 465-468. (In Russian)

19. Petrushev, P.; Popov, V. Rational Approximation of Real Functions; Cambridge University Press: Cambridge, UK, 1987.

20. Ivanov, K.; Totik, V. Fast Decreasing Polynomials. Constr. Approx. 1990, 6, 1-20. [CrossRef]

21. Kyurkchiev, N.; Andreev, A. Hausdorff approximation of functions different from zero at one point-implementation in programming environment MATHEMATICA. Serdica J. Comput. 2013, 7, 135-142.

22. Kyurkchiev, N.; Andreev, A. Synthesis of slot aerial grids with Hausdorff-type directive patterns-implementation in programming environment Mathematica. CR Acad. Bulg. Sci. 2013, 66, 1521-1528.

23. Kyurkchiev, N. Synthesis of Slot Aerial Grids with Hausdorff Type Directive Patterns. Ph.D. Thesis, VMEI, Sofia, Bulgaria, 1979. (In Bulgarian)

24. Sendov, Bl.; Schinev, H.; Kjurkchiev, N. Hausdorff-synthesis of aerial grids in scanning the directive diagram. Electropromishlenost Prib. 1984, 16, 203-205. (In Bulgarian)

25. Schinev, H.; Kjurkchiev, N.; Gachev, M. Experimental investigations of slot aerial grids with Hausdorff type directive patterns. Electropromishlenost Prib. 1979, 14, 223-224. (In Bulgarian)

26. Shinev, H.; Kyurkchiev, N.; Gachev, M.; Markov, S. Application of a class of polynomials of best approximation to linear antenna array synthesis. Izv. Vmei Sofia 1975, 34, 1-6. (In Bulgarian)

27. Kyurkchiev, N.; Sendov, Bl. Approximation of a class of functions by algebraic polynomials with respect to Hausdorff distance. Ann. Univ. Sofia Fac. Math. 1975, 67, 573-579. (In Bulgarian)

28. Appostolov, P. General theory, approximation method and design of electrical filters based on Hausdorff polynomials. Mech. Transp. Commun. 2007, 2, 1-8. (In Bulgarian)

29. Kyurkchiev, N.; Iliev, A.; Rahnev, A. A Look at the New Logistic Models with "Polynomial Variable Transfer"; Lap Lambert Academic Publishing: Saarbrucken, Germany, 2020.

30. Kyurkchiev, N.; Iliev, A.; Golev, A.; Rahnev, A. Some Non-standard Models in "Debugging and Test Theory" (Part 4); Plovdiv University Press: Plovdiv, Bulgaria, 2020.

31. Costarelli, D.; Vinti, G. Pointwise and uniform approximation by multivariate neural network operators of the max-product type. Neural Netw. 2016, 81, 81-90. [CrossRef]

32. Guliyev, N.; Ismailov, V. A single hidden layer feed forward network with only one neuron in the hidden layer san approximate any univariate function. Neural Comput. 2016, 28, 1289-1304. 
33. Iris, C.; Pacino, D.; Ropke, S.; Larsen, A. Integrated berth allocation and quay crane assignment problem: Set partitioning models and computational results. Transp. Res. Part Logist. Transp. Rev. 2015, 81, 75-97. [CrossRef]

34. Mauri, G. R.; Ribeiro, G. M.; Lorena, L. A. N.; Laporte, G. An adaptive large neighborhood search for the discrete and continuous berth allocation problem. Comput. Oper. Res. 2016 70, 140-154. [CrossRef]

35. Kyurkchiev, N. A new class of activation functions. Some related problems and applications. Biomath 2020, 9, 10. [CrossRef]

36. Kyurkchiev, N.; Iliev, A.; Markov, S. Some Techniques for Recurrence Generating of Activation Functions: Some Modeling and Approximation Aspects; Lap Lambert Academic Publishing: Saarbrucken, Germany, 2017.

37. Iliev, A.; Kyurkchiev, N.; Markov, S. A Note on the New Activation Function of Gompertz Type. Biomath Commun. 2017, 4, 20. [CrossRef]

38. Kyurkchiev, N.; Iliev, A.; Rahnev, A. A new class of activation functions based on the correcting amendments of Gompertz-Makeham type. Dyn. Syst. Appl. 2019, 28, 243-257. [CrossRef]

39. Kyurkchiev, N. Comments on the Yun's algebraic activation function. Some extensions in the trigonometric case. Dyn. Syst. Appl. 2019, 28, 533-543.

Publisher's Note: MDPI stays neutral with regard to jurisdictional claims in published maps and institutional affiliations.

(C) 2020 by the author. Licensee MDPI, Basel, Switzerland. This article is an open access article distributed under the terms and conditions of the Creative Commons Attribution (CC BY) license (http:// creativecommons.org/licenses/by/4.0/). 